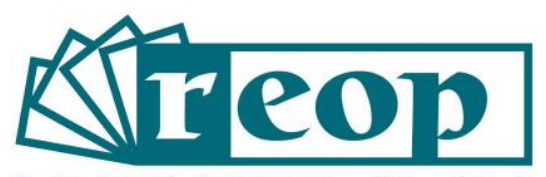

Revista Española de Orientación y Psicopedagogía

\title{
SERVICIOS UNIVERSITARIOS DE ATENCIÓN AL ALUMNADO CON DISCAPACIDAD EN ESPAÑA
}

\author{
UNIVERSITY SERVICES FOR STUDENTS WITH DISABILITIES IN SPAIN
}

\author{
Ana María Porto Castro \\ Universidad de Santiago de Compostela. Facultad de Ciencias de la Educación. Departamento de \\ Pedagogía y Didáctica. Santiago de Compostela, España \\ Enelina María Gerpe Pérez ${ }^{1}$ \\ Universidad de Santiago de Compostela. Facultad de Ciencias de la Educación. Departamento de \\ Pedagogía y Didáctica. Santiago de Compostela, España
}

\section{RESUMEN}

Los servicios de atención al alumnado con discapacidad disponibles en la mayoría de las universidades españolas comparten líneas de actuación comunes centradas en la eliminación de las barreras arquitectónicas, en la mejora de la accesibilidad, el asesoramiento, la orientación y la formación e información a toda la comunidad universitaria. En este trabajo, de carácter descriptivo, se pretendía conocer la situación actual de estos servicios, para ayudar a las instituciones universitarias a establecer nuevos objetivos y acciones a incorporar en sus políticas educativas y organizativas y a reforzar los procesos de orientación y tutoría antes, durante y después del ingreso en la universidad. Para ello se recopiló, describió y analizó la situación actual de los servicios de atención al alumnado con discapacidad de 50 universidades públicas y 26 privadas españolas. La recogida de información se llevó a cabo utilizando una hoja de registro diseñada ad hoc para el estudio. Los principales resultados muestran que, en la mayoría de las universidades analizadas, tanto públicas como privadas, se cuenta con un servicio o unidad de atención al alumnado con discapacidad; estos servicios son heterogéneos en su denominación, dependencia y actuaciones; en ellos se procura garantizar la igualdad de oportunidades y la inclusión del alumnado con discapacidad y los principales ámbitos de intervención se centran en la orientación, y el apoyo técnico y pedagógico. Por último, cabe señalar que una de las líneas de intervención

${ }^{1}$ Correspondencia: Enelina María Gerpe Pérez. Correo-e: emaria.gerpe@usc.es 
prioritarias es la sensibilización, la información y formación a toda la comunidad universitaria y la atención individualizada.

Palabras clave: Discapacidad, inclusión, orientación, servicios universitarios, tutoría.

\begin{abstract}
The services or units that cater for students with disabilities, available in most Spanish universities, share common lines of action, focused on the removal of architectural barriers, on the improvement of accessibility, advice, guidance, and training and information to the entire university community. The descriptive study reported in this paper purported to know and analyze the current situation of these services, to help university institutions to establish new objectives and actions to be incorporated into their educational and organizational policies and to reinforce guidance and mentoring processes before, during and after entering university. For this purpose, the current situation of the services of attention to students with disabilities of 50 public and 26 private Spanish universities was compiled, described, and analyzed. The information collection was carried out using a record sheet designed ad hoc for the study. The main results show that in most of the universities analyzed, both public and private, there is a service or unit that caters for students with disabilities; these services are heterogeneous in their name, dependence, and actions; they seek to guarantee equal opportunities and inclusion of students with disabilities, and the main areas of intervention focus on guidance, and technical and pedagogical support. Finally, it should be noted that one of the priority lines of intervention is awareness raising, information and training for the entire university community and individualized attention.
\end{abstract}

Key Words: Disability, guidance, inclusion, tutorial, university services.

\title{
Cómo citar este artículo:
}

Porto Castro, A. Ma., y Gerpe Pérez, E. Ma . (2020). Servicios universitarios de atención al alumnado con discapacidad en España. Revista Española de Orientación y Psicopedagogía, 31(3), 149-169. https://doi.org/10.5944/reop.vol.31.num.3.2020.29266

\section{Introducción}

La presencia de alumnado con discapacidad en las aulas universitarias es cada vez mayor (Abad Álvarez y Castro de Paz, 2008; Alcedo, Aguado, Real, González y Rueda 2007; Álvarez, Alegre y López, 2012; Hernández, Fernández, Carrión y Avilés, 2019; De Los Santos, Kupezynski y Mundy, 2019; Melero, Moriña y Perera, 2019), debido al éxito de las actuaciones y programas desarrollados en las etapas educativas anteriores (Bilbao, 2010) y a las políticas legislativas (Galán-Mañas, 2015) implementadas en las últimas décadas en torno a esta realidad. 
La Ley 137/1982, de 7 de abril, de integración social de los minusválidos (LISMI) estableció los principios básicos de atención a las personas con discapacidad en todos los ámbitos, entre ellos el educativo. Esta Ley se actualizará posteriormente con el Real Decreto Legislativo 1/2013, de 29 de noviembre por el que se aprueba el Texto Refundido de la Ley General de Los Derechos de las Personas con Discapacidad y su Inclusión Social.

En el marco de los estudios universitarios, el Real Decreto 743/2003, de 20 de junio, que regula la prueba de acceso a la universidad de los mayores de 25 años, establece en su artículo 5 que para los/as candidatos/as que en el momento de su inscripción justifiquen debidamente alguna discapacidad que les impida realizar la prueba de acceso con los medios ordinarios, se adoptarán las medidas oportunas para que puedan hacerla en las condiciones más favorables en función de su discapacidad. En el mismo año, el Real Decreto 1742/2003, de 19 de diciembre, establece la normativa básica para el acceso a los estudios universitarios de carácter oficial y señala, en el artículo 14, que las universidades reservarán un 3\% de las plazas disponibles para estudiantes que tengan reconocido un grado de minusvalía igual o superior al 33\%, y para aquellos con necesidades educativas especiales permanentes asociadas a circunstancias personales de discapacidad que durante su escolarización anterior precisen de recursos y apoyos para su plena normalización educativa.

La Ley 51/2003, de 2 de diciembre, de igualdad de oportunidades, no discriminación y accesibilidad universal de las personas con discapacidad (LIONDAU) constituye el marco legislativo que incluye la obligación de prestar atención a las personas con discapacidad y garantizar su acceso y permanencia en los estudios universitarios, además de señalar los cambios fundamentales en la accesibilidad, instalaciones y servicios universitarios, cuestiones formativas sobre la discapacidad en las titulaciones y su consideración como integrantes de pleno derecho en la comunidad universitaria.

Asimismo, la Ley 39/2006, de 14 de diciembre, de Promoción de la Autonomía Personal y Atención a las personas en situación de dependencia, regula las condiciones básicas que garantizan la igualdad en el ejercicio subjetivo de la ciudadanía, la promoción de la autonomía personal y la atención a las personas en situación de dependencia. Hay también que citar la Convención Internacional de los Derechos de las Personas con Discapacidad (ONU, 2006), cuyo fin es garantizar la promoción, protección y pleno disfrute de las personas con discapacidad, y hacer que gocen de plena igualdad ante la ley.

En 2007, la Ley Orgánica 4/2007, de 12 de abril, que modifica la Ley Orgánica 6/2001, de 21 de diciembre, de Universidades, establece una serie de programas y medidas ausentes en la ley anterior, de modo que se garantice el acceso a la universidad de las personas con algún tipo de discapacidad y se prohíba todo tipo de discriminación.

De igual modo, la Ley 49/2007, de 26 de diciembre, por la que se determina el régimen de infracciones y sanciones en materia de igualdad de oportunidades, no discriminación y accesibilidad universal de las personas con discapacidad, en su Exposición de motivos sustituye los términos "disminuidos", "minusválidos" o "deficientes" por "personas con discapacidad o con déficit de independencia" (Ley derogada por el Real Decreto Legislativo 1/2013 en la que se establecen los derechos de las personas con discapacidad y de su inclusión social).

En este mismo año, el Ministerio de Trabajo y Asuntos Sociales publica el Libro Blanco sobre Universidad y Discapacidad (Peralta, 2007) que describe y analiza la situación del alumnado universitario con discapacidad, identifica deficiencias y buenas prácticas y propone acciones de mejora para garantizar la igualdad de oportunidades y accesibilidad de los/as estudiantes con discapacidad en la universidad; el Real Decreto 1393/2007, de 29 de octubre, establece la ordenación de las enseñanzas universitarias oficiales, incidiendo en que las universidades deben disponer de servicios de apoyo y asesoramiento adecuados, cuya tarea debe ser la evaluación de las necesidades, y la realización de adaptaciones curriculares e itinerarios y estudios alternativos 
para los/as estudiantes con necesidades educativas específicas derivadas de una situación de discapacidad.

En esta línea, el Real Decreto 1791/2010, de 30 de diciembre, que aprueba el Estatuto del Estudiante Universitario, reconoce los derechos de las personas con discapacidad, reforzando los principios de igualdad de oportunidades y no discriminación en los distintos aspectos de la vida universitaria: académicos, deportivos, de participación estudiantil, movilidad, prácticas externas, becas y ayudas o actividades solidarias, y promueve la creación de servicios de atención a la comunidad universitaria con discapacidad (artículo 65.6); su artículo 7 recoge el derecho del alumnado universitario a la información y orientación vocacional, académica y profesional, y al asesoramiento por las universidades sobre las actividades de las mismas que les afecten (1.f); y el artículo 22 incide en que los programas y las actividades de tutoría deberán adaptarse a las necesidades de los estudiantes con discapacidad, procediendo los departamentos o centros, bajo la coordinación y supervisión de la unidad competente en cada Universidad, a las adaptaciones metodológicas precisas y, en su caso, al establecimiento de tutorías específicas en función de sus necesidades (1); y se promoverá el establecimiento de programas de tutoría permanente para que el estudiante con discapacidad pueda disponer de un profesor tutor a lo largo de sus estudios (2).

En este contexto cambiante, las universidades han evolucionado para dar respuesta a las necesidades del alumnado con discapacidad que llega a sus aulas, de tal modo que, si a principios de 1990 muy pocos concluían sus estudios universitarios, en la actualidad parece impensable que las universidades no dispongan de servicios, programas y especialistas que trabajen para garantizar el acceso, desarrollo y conclusión de los estudios universitarios de este colectivo.

Al amparo de esta normativa, gran parte de las universidades españolas han elaborado planes globales destinados al alumnado con discapacidad, desarrollado guías para orientar y apoyar al profesorado, diseñado e implementado programas de intervención y tutoría y creado servicios de apoyo (García-Cano, Castillejo, Jiménez y Martínez, 2017; Molina y González-Badía, 2006: Suárez y Castillo, 2020; Vieira y Ferreira, 2011), destacando el papel que adquieren los procesos de orientación y tutoría antes y después del ingreso a la universidad (Gairin et al, 2009). De acuerdo con Galán-Mañas, en las universidades españolas, a lo largo de los últimos años, los servicios de apoyo a las personas con discapacidad se han extendido considerablemente (2015). EI IV Estudio Universidad y Discapacidad (Fundación Universia, 2018) concluye que, de la totalidad de universidades españolas, 72 cuentan con un servicio de atención al alumnado con discapacidad, dependiente en su mayoría del Vicerrectorado de Estudiantes; el $76 \%$ desarrolla programas y acciones dirigidas al alumnado con discapacidad próximo a ingresar en la universidad; un $90 \%$ realiza acciones de tutorización y/o seguimiento de los estudiantes con discapacidad y un $68 \%$ realiza orientación psicoeducativa; en el $74 \%$ de los casos la labor de voluntariado se reconoce con créditos formativos, y aproximadamente las tres cuartas partes de estos servicios desarrollan programas y acciones orientadas a los/as futuros/as universitarios/as con discapacidad.

Los estudios desarrollados por Abad et al. (2008) y Alcantud, Ávila y Asensi (2000) señalan que las unidades o servicios universitarios destinadas a la atención del alumnado con discapacidad comparten líneas de actuación comunes, centradas en la eliminación de las barreras arquitectónicas y la mejora de la accesibilidad, la facilitación de la movilidad y el transporte, la elaboración de censos, el apoyo al estudio, la sensibilización de la comunidad universitaria, el apoyo técnico, las ayudas económicas, la gratuidad de matrícula, la formación del personal de administración y servicios y del personal docente e investigador, las adaptaciones curriculares y las políticas de inserción laboral.

Aunque los formatos y modelos de gestión de los servicios son diferentes entre sí (Comes, Parera, Vedriel y Vives, 2011; Molina y González-Badía, 2006), tanto en su origen como en su estructura (Vieira y Ferreira, 2011), prácticamente todos se ocupan de ofrecer información sobre 
acogida, asesoramiento, apoyo y atención personalizada; servicio de transporte adaptado; supresión de barreras arquitectónicas; ayudas técnicas; sensibilización y formación a toda la comunidad universitaria y coordinación con otros organismos e instituciones externas.

Del mismo modo, Núñez (2017) señala que los servicios de apoyo al alumnado con discapacidad en la universidad son muy heterogéneos en su estructura, profesionales que los integran, organización y recursos con que cuentan y que, en general, su actuación se orienta a la superación de barreras de acceso espaciales y materiales, pero no tanto a la intervención en cuestiones curriculares.

Dada la disparidad de los servicios de atención al alumnado con discapacidad y la diversidad de acciones que realizan, parece útil llevar a cabo una revisión de los mismos y un análisis de sus actuaciones para obtener una visión del conjunto de las universidades del Estado y cómo llevan a cabo la atención y orientación al alumnado con discapacidad que ingresa en sus aulas. El objetivo que se persigue en este artículo es recopilar, describir y analizar su situación actual. Conocer sus características y tendencias puede ayudar a las instituciones universitarias a establecer nuevos objetivos y acciones a incorporar en sus políticas educativas y organizativas y a reforzar los procesos de orientación y tutoría antes, durante y después del ingreso en la universidad.

\section{Método}

El estudio, de carácter descriptivo, pretende aportar información actualizada sobre los servicios de atención al alumnado con discapacidad disponibles en las universidades españolas, ofreciendo una panorámica global que permita conocer como se pone en práctica la ordenación de las enseñanzas universitarias oficiales en relación a estos servicios de apoyo y asesoramiento, los aspectos básicos que caracterizan su funcionamiento, y las actividades de apoyo, prevención, información, asesoramiento y orientación que realizan.

\section{Muestra}

El trabajo se llevó a cabo durante el curso académico 2018-2019. Se tomaron como objeto de análisis la totalidad de universidades españolas públicas y privadas, y más concretamente, sus páginas Web institucionales en lo correspondiente a los servicios de atención al alumnado con discapacidad. La muestra de la que se obtuvo información fue de 76 servicios, 50 de universidades públicas y 26 de privadas, distribuidas en las distintas Comunidades Autónomas del Estado, representando un $65.78 \%$ y un $34.21 \%$ del total de universidades respectivamente.

\section{Variables}

Las variables que analizar, siguiendo la línea de otros trabajos (Comes et al., 2011; Fundación Universia, 2018; Molina y González-Badía, 2006) son las siguientes: denominación del servicio; año de creación; dependencia orgánica dentro de la institución; finalidad; actuaciones; programas desarrollados; tipo de universidad (pública versus privada); Comunidad Autónoma en la que se 
ubica la universidad a la que pertenece el servicio. Se tomaron estas variables como objeto de estudio para poder profundizar en aquellos aspectos que pueden resultar relevantes respecto al funcionamiento de los servicios y la articulación de la intervención que realizan.

\section{Instrumentos y/o técnicas}

La recogida de información se realizó mediante una hoja de registro diseñada ad hoc para este trabajo. En ella se recogió directamente la información disponible en las páginas Web de las universidades referida al servicio de atención al alumnado con discapacidad, para su análisis posterior. Los aspectos registrados fueron los siguientes: Características descriptivas del servicio (creación, dependencia orgánica, denominación); Finalidad del servicio; Programas y Actuaciones.

\section{Procedimiento}

Se tomó como fuente de información la página Web de las universidades españolas, tanto públicas como privadas. Al inicio del curso, en el mes de octubre, se realizó una búsqueda y análisis de la información contenida en cada Web institucional. Posteriormente, a final de curso, concretamente en el mes de mayo, se efectuó una segunda revisión para actualizar y comprobar posibles cambios en la información recogida.

\section{Resultados}

A continuación, se presentan los resultados organizados atendiendo a las variables analizadas, el tipo de universidad (pública o privada) y la Comunidad Autónoma.

\section{Denominación del servicio}

En la siguiente tabla se observa que cada universidad pública opta por una denominación específica: centro (4), unidad (14), servicio (21), oficina (6), gabinete (1), área (3) o sistema de orientación (1), siendo las más frecuentes servicio y unidad. En catorce universidades privadas se designa como servicio de atención, de apoyo, o asesoramiento y en siete como unidad de apoyo o de atención al estudiante; algunos servicios incluyen los términos orientación psicológica, orientación educativa u orientación social. La mayoría añade el término apoyo, atención, asesoramiento o información; a la denominación servicio y sistema le acompaña en algún caso el término orientación. 
Tabla 1

Denominación del servicio

\begin{tabular}{|c|c|c|c|c|}
\hline \multirow[t]{3}{*}{ Denominación } & \multicolumn{4}{|c|}{ Número de universidades } \\
\hline & \multicolumn{2}{|c|}{ Públicas } & \multicolumn{2}{|c|}{ Privadas } \\
\hline & $\mathrm{N}$ & $\%$ & $\mathrm{~N}$ & $\%$ \\
\hline $\begin{array}{l}\text { Centro de Atención a Usuarios/ a Estudiantes con } \\
\text { Discapacidad }\end{array}$ & 4 & 8.00 & - & - \\
\hline $\begin{array}{l}\text { Unidad de Apoyo/Unidades de Atención al Estudiante } \\
\text { con Discapacidad (o términos afines) / Unidad de } \\
\text { Atención a la Discapacidad/ Unidad de Diversidad y } \\
\text { Discapacidad/ Unidad de Discapacidad/ Unidad de } \\
\text { Gestión de Programas y Cooperación/ Unidad de } \\
\text { Integración y Coordinación de Políticas de Discapacidad/ } \\
\text { Unidad de Trabajo Social }\end{array}$ & 14 & 28.00 & 7 & 26.92 \\
\hline $\begin{array}{l}\text { Servicio de Educación Inclusiva/ de Atención a la } \\
\text { Diversidad// de Atención/apoyo/asesoramiento al } \\
\text { Estudiante (con discapacidad) / de Participación e } \\
\text { Integración Universitaria/de Atención/información a la } \\
\text { Comunidad Universitario/ de Orientación Universitaria y } \\
\text { Atención a la Discapacidad/ de Información y } \\
\text { Orientación/ de Atención/de Orientación Psicológica, } \\
\text { Educativa y Social/ Servicio de Atención a la } \\
\text { Discapacidad }\end{array}$ & 21 & 42.00 & 14 & 53.84 \\
\hline $\begin{array}{l}\text { Oficina de atención a la diversidad (o atención al } \\
\text { alumnado con discapacidad) / Oficina de Relaciones } \\
\text { Internacionales y Responsabilidad Social/ Oficina de } \\
\text { Voluntariado e Integración }\end{array}$ & 6 & 12.00 & - & - \\
\hline $\begin{array}{l}\text { Gabinete de Sostenibilidad e Igualdad de Oportunidades/ } \\
\text { Gabinete Pedagógico y Tutorial. Servicio de Alumnos y } \\
\text { Becas }\end{array}$ & 1 & 2.00 & 1 & 3.84 \\
\hline $\begin{array}{l}\text { Área de Atención a la Diversidad Funcional/ Área de } \\
\text { Atención a la Discapacidad/ Área de Atención a la } \\
\text { Discapacidad/ Área de Atención al Estudiante con } \\
\text { Discapacidad }\end{array}$ & 3 & 6.00 & 2 & 7.69 \\
\hline $\begin{array}{l}\text { Departamento de Acción Social y } \\
\text { Departamento de Desarrollo Universitario }\end{array}$ & - & - & 2 & 7.69 \\
\hline Sistema de Orientación & 1 & 2.00 & - & - \\
\hline
\end{tabular}

Fuente: Elaboración propia

\section{Año de creación del servicio ${ }^{2}$}

Con los datos recopilados se comprueba que en las universidades públicas, la mayoría de los servicios se pusieron en marcha en el año 2004 (Universidad Nacional de Educación a Distancia, Universidad de Cádiz, Universidad de Huelva, Universidad de Extremadura, Universidad de A Coruña y Universidad Pública de Navarra) y 2005 (Universidad de Jaén, Universidad de Málaga, Universidad de Pablo Olavide, Universidad de Oviedo, Universidad de Alcalá de Henares), como refleja la tabla 2.

La primera universidad pública que contó con este servicio fue la Autónoma de Barcelona (1989) y entre las últimas figuran la Universidad de Jaén, la Universidad de Málaga, la Universidad Pablo Olavide y la Universidad de Oviedo (2005).

2 En la tabla 2 no figura el año de creación de los servicios de todas las universidades públicas porque no están disponibles en las Web institucionales consultadas. Las universidades privadas no registran este dato en las Web institucionales consultadas. 


\section{Tabla 2}

Año de creación y número de servicios en las universidades públicas

\begin{tabular}{|c|c|c|}
\hline Año de creación & $\mathbf{N}$ & Universidad pública \\
\hline 1989 & 1 & Universidad Autónoma de Barcelona \\
\hline 1990 & 1 & Universidad de Valencia- Estudios Generales \\
\hline 1991 & 1 & Universidad Jaume I \\
\hline 1992 & 2 & Universidad de Granada; Universidad de Barcelona \\
\hline 1994 & 1 & Universidad Pompeu Fabra \\
\hline 1996 & 4 & $\begin{array}{l}\text { Universidad de Almería; Universidad de Cantabria; Universidad Carlos III; } \\
\text { Universidad Politécnica de Valencia }\end{array}$ \\
\hline 1997 & 4 & $\begin{array}{l}\text { Universidad de Valladolid; Universidad Miguel Hernández de Elche; } \\
\text { Universidad de Extremadura; Universidad de Santiago de Compostela }\end{array}$ \\
\hline 1999 & 4 & $\begin{array}{l}\text { Universidad de La Laguna; Universidad de Alicante; Universidad de Islas } \\
\text { Baleares; Universidad de Sevilla }\end{array}$ \\
\hline 2000 & 1 & Universidad Politécnica de Cataluña \\
\hline 2001 & 2 & Universidad de Castilla La Mancha; Universidad del País Vasco \\
\hline 2002 & 4 & $\begin{array}{l}\text { Universidad de Burgos; Universidad de León; Universidad de Salamanca; } \\
\text { Universidad Autónoma de Madrid }\end{array}$ \\
\hline 2003 & 2 & Universidad Complutense de Madrid; Universidad Rey Juan Carlos \\
\hline 2004 & 6 & $\begin{array}{l}\text { Universidad de Huelva; Universidad de Extremadura; Universidad de A } \\
\text { Coruña; Universidad Pública de Navarra }\end{array}$ \\
\hline 2005 & 5 & $\begin{array}{l}\text { Universidad de Jaén; Universidad de Málaga; Universidad de Pablo Olavide; } \\
\text { Universidad de Oviedo; Universidad de Alcalá de Henares }\end{array}$ \\
\hline
\end{tabular}

Fuente: Elaboración propia

Dependencia orgánica en la institución universitaria

La dependencia orgánica es muy heterogénea. En este sentido, como se observa en la siguiente tabla, en la mayoría de las universidades públicas (25) el servicio depende del Vicerrectorado de Estudiantes (o términos afines); en cuatro del Servicio de Asistencia a la Comunidad Universitaria; en tres del Vicerrectorado para la Comunidad Universitaria y en dos del Servicio de Asuntos Sociales (Acción Social), de la Dirección General de Acción Solidaria, del Vicerrectorado de Campus y Calidad Ambiental, del Área de Accesibilidad o del Área de Discapacidad; no especifican esta información dos casos.

En las universidades privadas 8 servicios dependen del Vicerrectorado o Secretariado de Estudiantes, 1 del Gabinete de Comunicación y en 17 la WEB no especifica la dependencia institucional. 
Tabla 3

Dependencia orgánica del servicio en la universidad

\begin{tabular}{|c|c|c|c|c|}
\hline \multirow{3}{*}{ Dependencia } & \multicolumn{4}{|c|}{$\begin{array}{l}\text { Número de universidades } \\
\text { públicas }\end{array}$} \\
\hline & \multicolumn{2}{|c|}{ Públicas } & \multicolumn{2}{|c|}{ Privadas } \\
\hline & $\mathrm{N}$ & $\%$ & $\mathrm{~N}$ & $\%$ \\
\hline Vicerrectorado de Estudiantes (o términos afines) & 25 & 50.00 & - & - \\
\hline Servicio de Asuntos Sociales (acción social) & 2 & 4.00 & - & - \\
\hline Dirección General de Acción Solidaria & 2 & 4.00 & - & - \\
\hline Secretariado para la Inclusión y la Diversidad & 1 & 2.00 & - & - \\
\hline Servicio de Asistencia a la Comunidad Universitaria & 4 & 8.00 & - & - \\
\hline Vicerrectorado de Políticas Inclusivas y Vida Universitaria & 1 & 2.00 & - & - \\
\hline Vicerrectorado de Cultura y Participación social & 1 & 2.00 & - & - \\
\hline $\begin{array}{l}\text { Vicerrectorado de Acción Transversal y Cooperación con la } \\
\text { empresa }\end{array}$ & 1 & 2.00 & - & - \\
\hline $\begin{array}{l}\text { Vicerrectorado de Política Social, Calidad Ambiental y } \\
\text { Universidad Saludable }\end{array}$ & 1 & 2.00 & - & - \\
\hline Vicerrectorado de Campus y Calidad Ambiental & 2 & 4.00 & - & - \\
\hline $\begin{array}{l}\text { Vicerrectorado de Extensión Universitaria y Relación con la } \\
\text { Sociedad }\end{array}$ & 1 & 2.00 & - & - \\
\hline Área de Accesibilidad o Área de Discapacidad & 2 & 4.00 & - & - \\
\hline Vicerrectorado de Calidad y Dinamización Europea & 1 & 2.00 & - & - \\
\hline Vicerrectorado de Comunicación, Cultura y Servicios & 1 & 2.00 & - & - \\
\hline Vicerrectorado para la Comunidad Universitaria & 3 & 6.00 & - & - \\
\hline Gabinete de Comunicación & - & - & 1 & 3.84 \\
\hline $\begin{array}{l}\text { Secretariado/Vicerrectorado de Estudiantes (o términos } \\
\text { afines) }\end{array}$ & - & - & 8 & 30.76 \\
\hline Sin especificar & 2 & 4.00 & 17 & 65.38 \\
\hline
\end{tabular}

Fuente: Elaboración propia

Finalidad del Servicio

Todo servicio de atención al alumnado con discapacidad es, en palabras de Cotán (2017, p.57) "un apoyo que atiende sus demandas y necesidades, y ofrece recursos técnicos, adaptaciones curriculares y apoyo personal".

Tabla 4

Finalidad de los servicios de las universidades públicas ${ }^{3}$

\begin{tabular}{lcl}
\hline \multicolumn{1}{c}{ Comunidad Autónoma } & \multicolumn{1}{c}{ Universidades públicas } \\
\hline $\begin{array}{l}\text { Comunidad Autónoma de } \\
\text { Andalucía }\end{array}$ & $\begin{array}{c}\text { Universidad } \\
\text { de Almería }\end{array}$ & $\begin{array}{l}\text { Responder a las necesidades de orientación y apoyo } \\
\text { que alumnos/as y profesores/as presentan durante su } \\
\text { permanencia en la universidad. }\end{array}$ \\
\cline { 2 - 3 } & $\begin{array}{c}\text { Universidad } \\
\text { de Cádiz }\end{array}$ & $\begin{array}{l}\text { Garantizar un tratamiento equitativo y una efectiva } \\
\text { igualdad de oportunidades para cualquier miembro de } \\
\text { la Comunidad Universitaria. }\end{array}$ \\
\hline
\end{tabular}

Fuente: Elaboración propia

\footnotetext{
${ }^{3}$ Es necesario indicar que en aquellos casos donde no se especifican todas las universidades, tanto públicas como privadas y sus respectivas finalidades, se debe a que en las páginas WEB institucionales no aparece recogida dicha información.
} 
Tabla 4 (Continuación)

Finalidad de los servicios de las universidades públicas ${ }^{4}$

\begin{tabular}{|c|c|c|}
\hline Comunidad Autónoma & & Universidades públicas \\
\hline \multirow[t]{7}{*}{$\begin{array}{l}\text { Comunidad Autónoma de } \\
\text { Andalucía }\end{array}$} & $\begin{array}{l}\text { Universidad } \\
\text { de Córdoba }\end{array}$ & $\begin{array}{l}\text { Garantizar la igualdad de oportunidades y no } \\
\text { discriminación entre los miembros de la comunidad } \\
\text { universitaria, asumiendo la responsabilidad de atender } \\
\text { las situaciones de desigualdad y discriminación que } \\
\text { afectan a la convivencia, revirtiéndolas en procesos de } \\
\text { inclusión. }\end{array}$ \\
\hline & $\begin{array}{l}\text { Universidad } \\
\text { de Granada }\end{array}$ & $\begin{array}{l}\text { Facilitar la accesibilidad al alumnado con discapacidad; } \\
\text { informar, orientar y asesorar a los/as estudiantes sobre } \\
\text { los derechos y recursos sociales existentes, coordinar } \\
\text { actuaciones con otras instituciones, y facilitar información } \\
\text { y asesoramiento en materia de alojamiento. }\end{array}$ \\
\hline & $\begin{array}{l}\text { Universidad } \\
\text { de Huelva }\end{array}$ & $\begin{array}{l}\text { Hacer real y efectivo el derecho a cursar estudios } \\
\text { universitarios en condiciones de igualdad, poniendo el } \\
\text { foco en las necesidades educativas, materiales, y en las } \\
\text { cuestiones personales o psicosociales. }\end{array}$ \\
\hline & $\begin{array}{l}\text { Universidad } \\
\text { de Málaga }\end{array}$ & $\begin{array}{l}\text { Orientar y atender al alumnado, fomentando la igualdad } \\
\text { de oportunidades y la inclusión en el ámbito universitario. }\end{array}$ \\
\hline & $\begin{array}{l}\text { Universidad } \\
\text { de Pablo } \\
\text { Olavide }\end{array}$ & $\begin{array}{l}\text { Garantizar la igualdad de oportunidades de las personas } \\
\text { con discapacidad, ofreciendo los medios, apoyos y } \\
\text { recursos necesarios que aseguren una igualdad real y } \\
\text { efectiva en el ámbito universitario; prestar atención, } \\
\text { asesoramiento y apoyo integral e individualizado a } \\
\text { cualquier demanda del alumnado, personal de } \\
\text { administración y servicios y personal docente e } \\
\text { investigador. }\end{array}$ \\
\hline & $\begin{array}{l}\text { Universidad } \\
\text { de Sevilla }\end{array}$ & $\begin{array}{l}\text { Responder a los retos derivados de la situación de } \\
\text { discapacidad; garantizar el apoyo en el acceso y } \\
\text { permanencia durante el período de estudio y posterior } \\
\text { inserción laboral, a partir de una atención integral y } \\
\text { personalizada. }\end{array}$ \\
\hline & $\begin{array}{l}\text { Universidad } \\
\text { Internacional } \\
\text { de Andalucía }\end{array}$ & \\
\hline
\end{tabular}

\section{Comunidad Autónoma de Aragón}

Universidad

de Zaragoza

Garantizar la igualdad de oportunidades a través de la plena inclusión de los/as estudiantes en la vida académica; promover la sensibilización y concienciación de toda la comunidad universitaria.

Eliminar las limitaciones y dificultades que impiden una

\section{Comunidad Autónoma de Asturias.}

Universidad de Oviedo participación igualitaria y el desarrollo personal, académico y profesional de toda la comunidad universitaria; promover los principios de inclusión, transversalidad, pluralidad, diversidad, igualdad de oportunidades y equidad.

Garantizar la igualdad de oportunidades y equidad; promover la integración y participación del alumnado con

\section{Comunidad Autónoma de Canarias}

Universidad de la Laguna discapacidad en la comunidad universitaria, favoreciendo su desarrollo académico y aprendizaje; apoyar la labor docente y tutorial con estudiantes inscritos en el Programa de Atención al Estudiante con Discapacidad, PAED.

\footnotetext{
${ }^{4}$ Es necesario indicar que en aquellos casos donde no se especifican todas las universidades, tanto públicas como privadas y sus respectivas finalidades, se debe a que en las páginas WEB institucionales no aparece recogida dicha información.
} 
Tabla 4 (Continuación)

Finalidad de los servicios de las universidades públicas ${ }^{5}$

\begin{tabular}{|c|c|c|}
\hline Comunidad Autónoma & & Universidades públicas \\
\hline $\begin{array}{l}\text { Comunidad Autónoma de } \\
\text { Cantabria }\end{array}$ & $\begin{array}{l}\text { Universidad } \\
\text { de Cantabria }\end{array}$ & $\begin{array}{l}\text { Hacer efectiva la igualdad de oportunidades en la } \\
\text { educación superior. }\end{array}$ \\
\hline $\begin{array}{l}\text { Comunidad Autónoma de } \\
\text { Castilla la Mancha }\end{array}$ & $\begin{array}{l}\text { Universidad } \\
\text { de Castilla La } \\
\text { Mancha }\end{array}$ & $\begin{array}{l}\text { Dar soporte al alumnado universitario con discapacidad } \\
\text { que lo solicite, garantizando la igualdad de condiciones y } \\
\text { oportunidades y la integración en todos los aspectos de } \\
\text { la vida académica. }\end{array}$ \\
\hline \multirow{4}{*}{$\begin{array}{l}\text { Comunidad Autónoma de } \\
\text { Castilla y León }\end{array}$} & $\begin{array}{l}\text { Universidad } \\
\text { de Burgos }\end{array}$ & $\begin{array}{l}\text { Garantizar la igualdad de oportunidades del alumnado } \\
\text { universitario con discapacidad. }\end{array}$ \\
\hline & $\begin{array}{l}\text { Universidad } \\
\text { de León }\end{array}$ & $\begin{array}{l}\text { Garantizar la igualdad de oportunidades de las personas } \\
\text { con discapacidad; promover la supresión de barreras en } \\
\text { el acceso, participación y aprendizaje;-realizar acciones } \\
\text { formativas que desarrollen la sensibilidad y solidaridad } \\
\text { en el ámbito universitario hacia las personas con } \\
\text { discapacidad. }\end{array}$ \\
\hline & $\begin{array}{l}\text { Universidad } \\
\text { de } \\
\text { Salamanca }\end{array}$ & $\begin{array}{l}\text { Facilitar el acceso y la promoción en la enseñanza } \\
\text { universitaria de las personas con discapacidad. }\end{array}$ \\
\hline & $\begin{array}{l}\text { Universidad } \\
\text { de Valladolid }\end{array}$ & $\begin{array}{l}\text { Facilitar la inclusión y la mayor autonomía posible del } \\
\text { alumnado con discapacidad en el contexto universitario. }\end{array}$ \\
\hline \multirow{4}{*}{$\begin{array}{l}\text { Comunidad Autónoma de } \\
\text { Cataluña }\end{array}$} & $\begin{array}{l}\text { Universidad } \\
\text { Autónoma de } \\
\text { Barcelona }\end{array}$ & $\begin{array}{l}\text { Garantizar el acceso a los estudios superiores en } \\
\text { igualdad de condiciones para poder disfrutar de una vida } \\
\text { académica y social plena y autónoma. }\end{array}$ \\
\hline & $\begin{array}{l}\text { Universidad } \\
\text { de Barcelona }\end{array}$ & $\begin{array}{l}\text { Garantizar un trato equitativo y la igualdad de } \\
\text { oportunidades efectiva en la vida académica } \\
\text { universitaria; sensibilizar y concienciar a toda la } \\
\text { comunidad universitaria y a la sociedad en general. }\end{array}$ \\
\hline & $\begin{array}{l}\text { Universidad } \\
\text { de Girona }\end{array}$ & $\begin{array}{l}\text { Orientar, asesorar y proporcionar recursos técnicos o } \\
\text { académicos a las personas con discapacidad o } \\
\text { necesidades educativas, en cuestiones relacionadas con } \\
\text { la participación en todos los ámbitos de la vida } \\
\text { universitaria. }\end{array}$ \\
\hline & $\begin{array}{l}\text { Universidad } \\
\text { de Lleida }\end{array}$ & $\begin{array}{l}\text { Informar, asesorar y orientar a los/as estudiantes, } \\
\text { comunidad universitaria y ciudadanía en general. }\end{array}$ \\
\hline \multirow{4}{*}{$\begin{array}{l}\text { Comunidad Autónoma de } \\
\text { Madrid }\end{array}$} & $\begin{array}{l}\text { Universidad } \\
\text { de Alcalá de } \\
\text { Henares }\end{array}$ & $\begin{array}{l}\text { Impulsar, desarrollar, coordinar y evaluar todas aquellas } \\
\text { actuaciones adoptadas en y desde la propia institución } \\
\text { que favorezcan la plena inclusión de las personas con } \\
\text { discapacidad en el ámbito universitario. }\end{array}$ \\
\hline & $\begin{array}{l}\text { Universidad } \\
\text { Autónoma de } \\
\text { Madrid }\end{array}$ & $\begin{array}{l}\text { Trabajar a favor de la igualdad de oportunidades y la } \\
\text { plena inclusión del alumnado con necesidades } \\
\text { educativas en la vida académica; sensibilizar y } \\
\text { concienciar a todos los miembros de la comunidad } \\
\text { universitaria. }\end{array}$ \\
\hline & $\begin{array}{l}\text { Universidad } \\
\text { Carlos III }\end{array}$ & $\begin{array}{l}\text { Solicitar adaptaciones y servicios destinados al } \\
\text { alumnado con discapacidad durante sus estudios } \\
\text { universitarios. }\end{array}$ \\
\hline & $\begin{array}{l}\text { Universidad } \\
\text { Complutense } \\
\text { de Madrid }\end{array}$ & $\begin{array}{l}\text { Atender, de modo personalizado, a todos los miembros } \\
\text { de la Comunidad Universitaria, lo que se concreta en el } \\
\text { apoyo, acompañamiento, escucha, orientación, } \\
\text { asistencia, formación y asesoramiento. }\end{array}$ \\
\hline
\end{tabular}

Fuente: Elaboración propia

\footnotetext{
${ }^{5}$ Es necesario indicar que en aquellos casos donde no se especifican todas las universidades, tanto públicas como privadas y sus respectivas finalidades, se debe a que en las páginas WEB institucionales no aparece recogida dicha información.
} 
Tabla 4 (Continuación)

Finalidad de los servicios de las universidades públicas ${ }^{6}$

\begin{tabular}{|c|c|c|}
\hline Comunidad Autón & ma & Universidades públicas \\
\hline \multirow[t]{3}{*}{$\begin{array}{l}\text { Comunidad } \\
\text { Autónoma de Madrid }\end{array}$} & $\begin{array}{l}\text { Universidad } \\
\text { Politécnica de } \\
\text { Madrid }\end{array}$ & $\begin{array}{l}\text { Garantizar la igualdad de oportunidades y no discriminación } \\
\text { en el acceso, permanencia y progreso en el ámbito } \\
\text { universitario de aquellos miembros de la Comunidad } \\
\text { Universitaria en situación de discapacidad; concienciar y } \\
\text { sensibilizar sobre la educación sin barreras y diseño para } \\
\text { todos/as. }\end{array}$ \\
\hline & $\begin{array}{l}\text { Universidad Rey } \\
\text { Juan Carlos }\end{array}$ & $\begin{array}{l}\text { Lograr la igualdad de oportunidades de todos los miembros } \\
\text { de la Universidad en el desarrollo de los estudios, o en el } \\
\text { desempeño laboral. }\end{array}$ \\
\hline & $\begin{array}{l}\text { Universidad } \\
\text { Nacional de } \\
\text { Educación a } \\
\text { Distancia (UNED) }\end{array}$ & $\begin{array}{l}\text { Proporcionar las mismas oportunidades que al resto de } \\
\text { estudiantes y contribuir a la supresión de las barreras en el } \\
\text { acceso, participación y aprendizaje de todas las personas } \\
\text { con discapacidad que integran la comunidad universitaria. }\end{array}$ \\
\hline \multirow{5}{*}{$\begin{array}{l}\text { Comunidad } \\
\text { Autónoma de } \\
\text { Valencia }\end{array}$} & $\begin{array}{l}\text { Universidad de } \\
\text { Alicante }\end{array}$ & $\begin{array}{l}\text { Garantizar la participación plena del alumnado con } \\
\text { discapacidad en el ámbito universitario. }\end{array}$ \\
\hline & $\begin{array}{l}\text { Universidad } \\
\text { Jaume I }\end{array}$ & $\begin{array}{l}\text { Prestar apoyo personalizado al sujeto y su entorno } \\
\text { académico; hacer de la universidad una institución } \\
\text { inclusiva; promover la sensibilización de la comunidad } \\
\text { universitaria. }\end{array}$ \\
\hline & $\begin{array}{l}\text { Universidad } \\
\text { Miguel Hernández } \\
\text { de Elche }\end{array}$ & $\begin{array}{l}\text { Garantizar la igualdad de oportunidades, apostando por la } \\
\text { inclusión. }\end{array}$ \\
\hline & $\begin{array}{l}\text { Universidad } \\
\text { Politécnica de } \\
\text { Valencia }\end{array}$ & $\begin{array}{l}\text { Apoyar y garantizar la igualdad de oportunidades en el } \\
\text { acceso, permanencia y posterior inserción laboral, } \\
\text { desarrollando planes personalizados de atención, } \\
\text { asesorando al personal docente e investigador en materia } \\
\text { de discapacidad, formando y sensibilizando a la comunidad } \\
\text { universitaria y desarrollando experiencias de empleo con } \\
\text { personas con discapacidad. }\end{array}$ \\
\hline & $\begin{array}{l}\text { Universidad de } \\
\text { Valencia- } \\
\text { Estudios } \\
\text { Generales }\end{array}$ & $\begin{array}{l}\text { Velar por el principio de igualdad de oportunidades y la no } \\
\text { discriminación. }\end{array}$ \\
\hline $\begin{array}{l}\text { Comunidad } \\
\text { Autónoma de } \\
\text { Extremadura }\end{array}$ & $\begin{array}{l}\text { Universidad de } \\
\text { Extremadura }\end{array}$ & $\begin{array}{l}\text { Garantizar la igualdad de oportunidades, la plena inclusión } \\
\text { de los/as estudiantes con discapacidad y/o necesidades } \\
\text { educativas procurando, la adaptación de los procesos de } \\
\text { enseñanza a sus características y necesidades. }\end{array}$ \\
\hline \multirow{3}{*}{$\begin{array}{l}\text { Comunidad } \\
\text { Autónoma de Galicia }\end{array}$} & $\begin{array}{l}\text { Universidad de A } \\
\text { Coruña }\end{array}$ & $\begin{array}{l}\text { Asesorar y sensibilizar a toda la comunidad universitaria } \\
\text { sobre derechos, vivencias y necesidades de las personas } \\
\text { con discapacidad, proporcionando atención individualizada, } \\
\text { apoyo, ayudas técnicas y redes de intercambio de } \\
\text { información; eliminar, de modo progresivo, las barreras } \\
\text { arquitectónicas y de comunicación de la institución. }\end{array}$ \\
\hline & $\begin{array}{l}\text { Universidad de } \\
\text { Santiago de } \\
\text { Compostela }\end{array}$ & $\begin{array}{l}\text { Coordinar la oferta de actividades de voluntariado y } \\
\text { participación social de la institución, que pretenden } \\
\text { favorecer la formación integral del alumnado. }\end{array}$ \\
\hline & $\begin{array}{l}\text { Universidad de } \\
\text { Vigo }\end{array}$ & $\begin{array}{l}\text { Integrar totalmente a las personas con necesidades } \\
\text { educativas específicas, tanto en el ámbito académico como } \\
\text { laboral. }\end{array}$ \\
\hline
\end{tabular}

Fuente: Elaboración propia

${ }^{6}$ Es necesario indicar que en aquellos casos donde no se especifican todas las universidades, tanto públicas como privadas y sus respectivas finalidades, se debe a que en las páginas WEB institucionales no aparece recogida dicha información. 
Tabla 4 (Continuación)

Finalidad de los servicios de las universidades públicas ${ }^{7}$

\begin{tabular}{lcl}
\hline \multicolumn{1}{c}{ Comunidad Autónoma } & \multicolumn{1}{c}{ Universidades públicas } \\
\hline $\begin{array}{l}\text { Comunidad Autónoma } \\
\text { de La Rioja }\end{array}$ & $\begin{array}{c}\text { Universidad de la la } \\
\text { Rioja }\end{array}$ & $\begin{array}{l}\text { alumnado con discapacidad, facilitando las adaptaciones } \\
\text { curriculares precisas, cuando se solicitan, siempre que } \\
\text { no afecten al contenido básico de los conocimientos } \\
\text { mínimos exigidos, los contenidos académicos, las } \\
\text { exigencias de dominio de competencias y habilidades y } \\
\text { no discriminen a ningún otro/a alumno/a. }\end{array}$ \\
\hline $\begin{array}{l}\text { Comunidad Autónoma } \\
\text { del País Vasco }\end{array}$ & $\begin{array}{c}\text { Universidad del } \\
\text { País Vasco }\end{array}$ & $\begin{array}{l}\text { Garantizar el principio de igualdad de oportunidades, } \\
\text { facilitando el acceso a los estudios, servicios y } \\
\text { participación plena en la institución universitaria. }\end{array}$ \\
\hline $\begin{array}{l}\text { Comunidad Autónoma } \\
\text { de Murcia }\end{array}$ & $\begin{array}{c}\text { Universidad de } \\
\text { Murcia respuesta a las necesidades de tipo social, personal, } \\
\text { y pedagógicas de la comunidad universitaria, sobre todo } \\
\text { de los/as estudiantes con discapacidad. }\end{array}$ \\
\hline $\begin{array}{l}\text { Comunidad Autónoma } \\
\text { de las Islas Baleares }\end{array}$ & $\begin{array}{c}\text { Universidad de las } \\
\text { Islas Baleares }\end{array}$ & \\
\hline $\begin{array}{c}\text { Comunidad Autónoma } \\
\text { de Navarra }\end{array}$ & $\begin{array}{c}\text { Universidad } \\
\text { Pública de } \\
\text { Navarra }\end{array}$ & \\
\hline
\end{tabular}

Fuente: Elaboración propia

Tabla 5

Finalidad de los servicios de las universidades privadas

\begin{tabular}{|c|c|c|}
\hline Comunidad Autónoma & & Universidades públicas \\
\hline $\begin{array}{l}\text { Comunidad Autónoma de } \\
\text { Andalucía }\end{array}$ & $\begin{array}{l}\text { Universidad } \\
\text { Loyola de } \\
\text { Andalucía }\end{array}$ & $\begin{array}{l}\text { Proporcionar a la comunidad universitaria, } \\
\text { especialmente al alumnado de grado, información, } \\
\text { asesoramiento y formación en los ámbitos personal, } \\
\text { social y académico. }\end{array}$ \\
\hline $\begin{array}{l}\text { Comunidad Autónoma de } \\
\text { Aragón }\end{array}$ & $\begin{array}{l}\text { Universidad } \\
\text { San Jorge }\end{array}$ & $\begin{array}{l}\text { Responder a las necesidades de las personas con } \\
\text { discapacidad, en igualdad de oportunidades, sin } \\
\text { disminuir la calidad académica, ni variar los contenidos } \\
\text { de la titulación. }\end{array}$ \\
\hline $\begin{array}{l}\text { Comunidad Autónoma de } \\
\text { Castilla y León }\end{array}$ & $\begin{array}{l}\text { Universidad } \\
\text { Europea } \\
\text { Miguel de } \\
\text { Cervantes }\end{array}$ & $\begin{array}{l}\text { Garantizar el pleno desarrollo e integración del } \\
\text { alumnado, facilitando su comunicación con los } \\
\text { diferentes servicios disponibles. }\end{array}$ \\
\hline $\begin{array}{l}\text { Comunidad Autónoma de } \\
\text { Cataluña }\end{array}$ & $\begin{array}{l}\text { Universidad } \\
\text { Abat Oliba } \\
\text { Ceu }\end{array}$ & $\begin{array}{l}\text { Atender las dificultades de aprendizaje y adaptación, } \\
\text { rendimiento académico y metodología de estudio; } \\
\text { asesorar personalmente y orientar curricularmente, } \\
\text { según el diagnóstico, bajo el respaldo de la acción } \\
\text { tutorial. }\end{array}$ \\
\hline & $\begin{array}{l}\text { Universidad } \\
\text { Ramón Llull }\end{array}$ & $\begin{array}{l}\text { Impulsar y coordinar acciones para lograr que la } \\
\text { comunidad universitaria desarrolle su actividad en } \\
\text { igualdad. }\end{array}$ \\
\hline Comunidad Autónoma de & $\begin{array}{l}\text { Universidad a } \\
\text { Distancia de } \\
\text { Madrid }\end{array}$ & $\begin{array}{l}\text { Trabajar para que las personas con discapacidad } \\
\text { tengan iguales oportunidades. }\end{array}$ \\
\hline & $\begin{array}{l}\text { Universidad } \\
\text { Alfonso X El } \\
\text { Sabio }\end{array}$ & $\begin{array}{l}\text { Proporcionar apoyo integral y personalizado y } \\
\text { orientación psicológica y pedagógica para lograr un } \\
\text { buen rendimiento académico. }\end{array}$ \\
\hline
\end{tabular}

Fuente: Elaboración propia 7 Es necesario indicar que en aquellos casos donde no se especifican todas las universidades, tanto públicas como privadas y sus
respectivas finalidades, se debe a que en las páginas WEB institucionales no aparece recogida dicha información. 


\section{Tabla 5}

Finalidad de los servicios de las universidades privadas

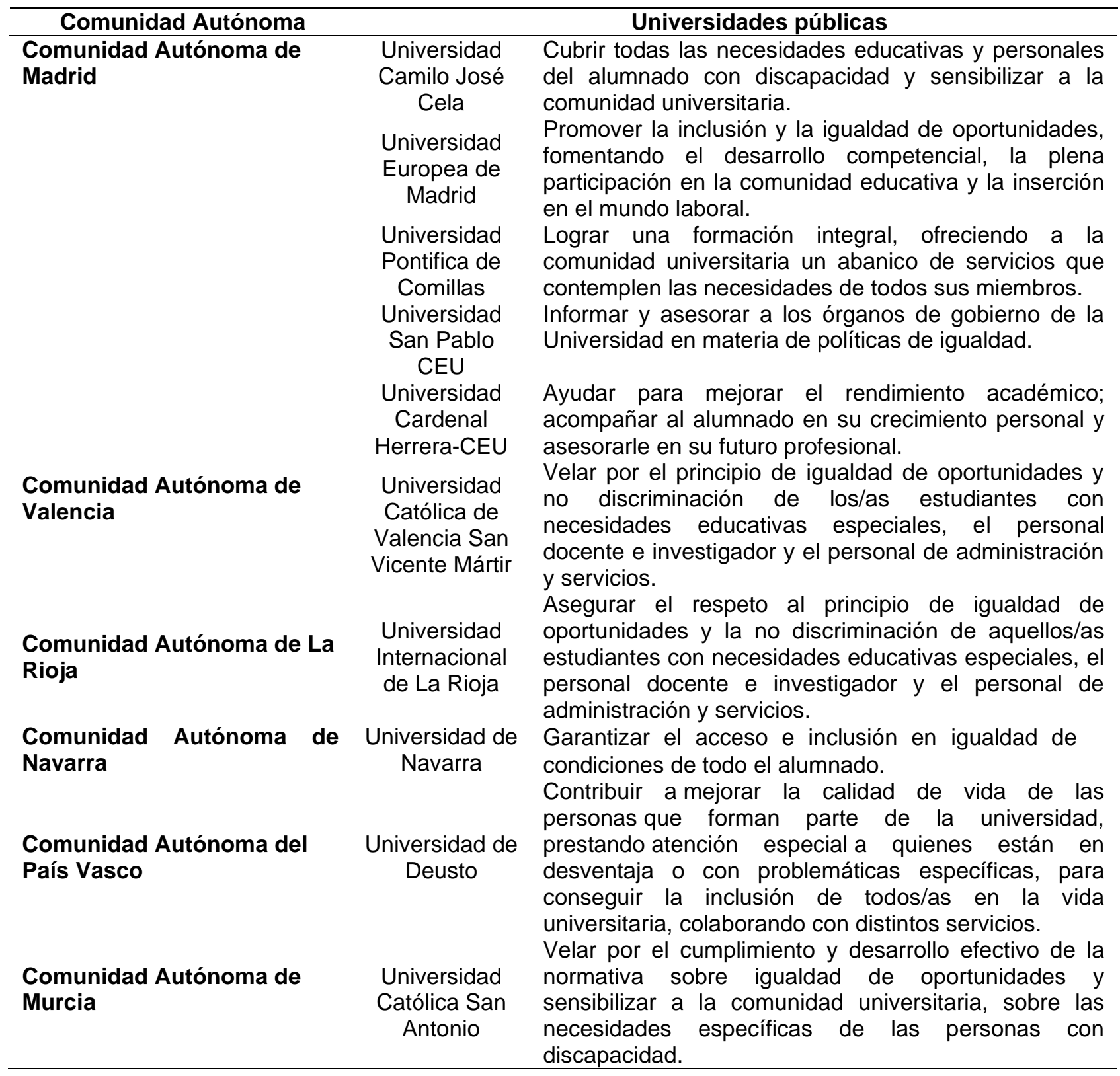

Fuente: Elaboración propia

Teniendo en cuenta que la mayoría de los servicios de las universidades analizadas forman parte de la Red de Servicios de Apoyo de Personas con discapacidad (Red SAPDU), encaminada a fomentar la colaboración entre los diferentes servicios de atención a los/as estudiantes con discapacidad; proponer actuaciones de carácter transversal en materia de discapacidad; promover medidas para el cumplimiento efectivo de lo regulado sobre discapacidad; desarrollar pautas que orienten la incorporación efectiva y activa de las personas con discapacidad en el conjunto de la vida universitaria, bajo la premisa de la igualdad de oportunidades, accesibilidad universal y diseño para todos, y la promoción, difusión e intercambio de buenas prácticas, se puede decir que, en líneas generales, en gran parte de las universidades objeto de estudio, tanto públicas como privadas, la finalidad principal del servicio de atención al alumnado con discapacidad es: garantizar la igualdad de oportunidades y la inclusión; sensibilizar y concienciar a toda la 
comunidad universitaria; informar, orientar y asesorar al alumnado, personal docente e investigador y personal de administración y servicios sobre los procesos de inclusión y las demandas del alumnado con discapacidad, y prestar atención y apoyo integral y personalizado a todos los miembros de la comunidad universitaria que lo precisen. Destacan pues los fines relacionados con el respeto al principio de igualdad de oportunidades, la atención individualizada y la orientación y asesoramiento, y también la sensibilización de toda la comunidad universitaria.

\section{Actuaciones}

Respecto a las actuaciones realizadas en los distintos servicios analizados, y dada su heterogeneidad, para facilitar su análisis e interpretación se han agrupado en diez bloques de contenido que atienden a las siguientes áreas de acción:

- Adaptación a las necesidades del alumnado con discapacidad. Incluye adaptaciones curriculares, de las pruebas de evaluación y de acceso a la universidad; adaptaciones para acomodar el espacio, por ejemplo, en las residencias universitarias.

- Accesibilidad. Se refiere a la supresión de barreras arquitectónicas y/o de cualquier otro tipo (por ejemplo, a los documentos de trabajo, etc.), y a la elaboración de planes de accesibilidad de la institución universitaria.

- Atención Individualizada. Recoge actuaciones relacionadas con la orientación, atención, seguimiento y acompañamiento individualizado y personalizado realizado antes, durante y después de la etapa universitaria; recepción y respuesta a las demandas y problemas; promoción de la autonomía personal; facilitación de asistencia a tutorías; atención psicológica y mediación.

- Apoyo educativo. Incluye aspectos relacionados con el asesoramiento educativo y al estudio; intermediación académica y facilitación del proceso de enseñanza y aprendizaje.

- Formación. Alude a la información, formación y apoyo al personal docente e investigador, personal de administración y servicios y alumnado en general, sobre la atención a la discapacidad y la inclusión.

- Apoyo técnico y económico. Referida a la gestión de ayudas económicas (por ejemplo, el bono-taxi); préstamo de dispositivos; transporte adaptado; reserva de plazas de aparcamiento; asistente personal, interprete de lenguaje de signos, tomador/a de apuntes; servicios complementarios (deporte, actividades culturales, etc.).

- Actuaciones en colaboración con otras entidades. Agrupa el diseño de actividades de colaboración con otros servicios de la propia universidad, y/o con asociaciones y entidades externas, y a las acciones relacionadas con el voluntariado.

- Actuaciones de sensibilización. Acciones destinadas a la promoción de actitudes positivas en la comunidad universitaria ante la discapacidad y la inclusión.

- Inserción laboral. Actuaciones para promover y facilitar la empleabilidad.

- Investigación. Referida a la promoción en la universidad de investigación relacionada con la temática. 
De los datos obtenidos sobre este punto se puede decir que, en líneas generales, prevalecen actuaciones vinculadas con la atención individualizada al estudiante con discapacidad.

En las universidades públicas también se proporciona apoyo técnico y económico (24), se realizan actuaciones para la mejora de la accesibilidad (22) y se procura la adaptación del currículo, la evaluación, los espacios, etc., a las necesidades del alumnado con discapacidad. Por el contrario, entre las actuaciones que menos se llevan a cabo se sitúan la investigación (1) o la colaboración con otras entidades (6).

Las actuaciones de los servicios de las universidades privadas se centran, sobre todo, en la sensibilización (12) y la adaptación a las necesidades del alumnado con discapacidad (10). Entre las de menor frecuencia están la inserción laboral y la colaboración con otras entidades (1 universidad en cada caso). Cabe señalar, finalmente, que no se da cuenta en ningún caso de actuaciones relacionadas con la investigación sobre discapacidad e inclusión en la educación superior.

\section{Tabla 6}

Actuaciones de los servicios

\begin{tabular}{lcc}
\hline \multicolumn{1}{c}{ Actuaciones } & \multicolumn{2}{c}{ Número de universidades } \\
\cline { 2 - 3 } & Públicas & Privadas \\
\hline Adaptación necesidades del alumnado con discapacidad & 21 & 10 \\
Accesibilidad & 22 & 6 \\
Atención individualizada & 36 & 19 \\
Apoyo educativo & 8 & 4 \\
Formación & 10 & 4 \\
Apoyo técnico y económico & 24 & 2 \\
Colaboración con otras entidades & 6 & 1 \\
Sensibilización & 15 & 11 \\
Inserción laboral & 5 & - \\
Investigación & 1 & - \\
\hline
\end{tabular}

Fuente: Elaboración propia

\section{Programas}

De acuerdo con Alcantud et al. (2000) los programas desarrollados en las universidades para atender al alumnado con discapacidad se ocupan de cuestiones como las barreras arquitectónicas y la eliminación o mejora de la accesibilidad, la facilitación del transporte y movilidad, la elaboración de censos, el apoyo técnico y al estudio, o la sensibilización a la comunidad, y todos persiguen como objetivo "La normalización de la vida universitaria de los alumnos con discapacidad" (p. 38).

En este artículo, se han agrupado los programas desarrollados en los servicios de atención al alumnado con discapacidad atendiendo a su tipología, en los siguientes:

- Programas de formación. Tratan aspectos relacionados con la formación, información y sensibilización de toda la comunidad educativa ante la discapacidad. También se centran en la promoción y el desarrollo de actitudes positivas hacia el alumnado con discapacidad y su inclusión en el contexto universitario.

- Programas de atención personalizada. Incluyen programas de asesoramiento individualizado, de orientación y atención tutorial, de atención psicológica y al estudio, de atención sociosanitaria y apoyo personal y de intermediación académica. 
- Programas de accesibilidad. Encaminados a la supresión de barreras en los diferentes contextos y ambientes en los que se desenvuelven las actividades de los/as alumnos/as con discapacidad durante sus estudios universitarios.

- Programas de Apoyo. Se ocupan de todo tipo de soportes o apoyos que facilitan el proceso de enseñanza y aprendizaje (apoyos técnicos, alumno-colaborador, promoción del voluntariado).

- Programas de inserción laboral. Encaminados a garantizar la incorporación al mundo laboral de las personas con discapacidad en igualdad de condiciones que el resto de sus compañeros/as.

- Programas de relación con otras instituciones. Tratan aspectos vinculados al fomento y desarrollo de acciones conjuntas con otras instituciones y organismos, de carácter público o privado.

- Programas de acogida. Destinados a asegurar que el proceso de incorporación a la universidad sea lo más natural posible y responda a todas las necesidades y demandas del alumnado.

- Otros programas, entre los que cabe citar aquellos relacionados con la actuación transversal y la intervención social.

Como se puede observar en la tabla 7, la mayoría de las universidades públicas dedican sus esfuerzos a diseñar programas de intervención relacionados con las ayudas al alumnado con discapacidad, la formación a la comunidad universitaria y la atención individualizada. Son menos las que a través de su servicio desarrollan programas de mejora de la accesibilidad o la colaboración con otras instituciones.

En las universidades privadas, los programas se centran, principalmente, en la formación y la atención individualizada y, mucho menos, en la inserción laboral o las ayudas. No se registran programas de accesibilidad, acogida o programas de relación con otras entidades.

\section{Tabla 7}

Programas desarrollados desde el servicio

\begin{tabular}{lcc}
\hline \multicolumn{1}{c}{ Programas } & \multicolumn{2}{c}{ Número de universidades } \\
\cline { 2 - 3 } & Públicas & Privadas \\
\hline Programas de formación & 12 & 4 \\
Programa de atención individualizada & 12 & 7 \\
Programas de mejora de la accesibilidad & 1 & - \\
Programa de apoyo & 13 & 1 \\
Programas de inserción laboral & 3 & 1 \\
Programas de relación con otras instituciones & 1 & - \\
Programas de acogida & 4 & - \\
Otros & 3 & - \\
\hline
\end{tabular}

Fuente: Elaboración propia 


\section{Conclusiones y Discusión}

En este trabajo se han revisado los servicios de atención al estudiante universitario con discapacidad correspondientes a la totalidad de universidades públicas y privadas españolas, lo que ha permitido presentar un panorama actualizado y amplio de esta realidad y afirmar que si bien el año 2005 solo un 53\% de las universidades españolas contaba con este servicio (Vieira y Ferreira, 2011), catorce años después, en 2019, un $98 \%$ de las universidades públicas y un $84,37 \%$ de las privadas existentes dispone de dicho servicio, confirmando la evolución numérica que se ha producido en los últimos años de estos servicios y la adecuación a la normativa vigente.

Respecto a la denominación, se constata que varía de una universidad a otra, si bien en la mayoría de los casos se hace referencia explícita a la especificidad del servicio al incluir términos como: centro de atención/unidad de atención/servicio de apoyo, a lo que se añade: estudiante/alumnado con discapacidad. Esto es así tanto en el caso de las universidades públicas como de las privadas. En cuanto a la dependencia orgánica, se ha podido comprobar que es también variada, aunque con una clara vinculación al Vicerrectorado de Estudiantes, dato coincidente con lo expuesto en otros trabajos como el de Alcantud et al. (2000) o el de Bausela Herreras (2002), si bien se observa que las actuaciones suelen también estar dirigidas a los demás miembros de la comunidad universitaria.

Paralelamente, y en consonancia con lo expuesto por Abad et al.(2008), se comprueba que las líneas de actuación aunque diversas se vinculan, principalmente, a la atención individualizada al estudiante con discapacidad tanto en las universidades públicas como en las privadas, el apoyo técnico y económico, la mejora de la accesibilidad y la sensibilización de toda la comunidad universitaria hacia la discapacidad, como apunta Galán - Mañas (2015), al señalar las principales actuaciones que desarrollan los servicios y unidades de atención al alumnado con discapacidad en la universidad.

Por lo que se refiere a la finalidad que en ellos se persigue, aún teniendo en cuenta las diferencias entre unos y otros servicios, en la mayoría de los casos se busca garantizar la igualdad de oportunidades y la inclusión, la orientación, asesoramiento y atención individualizada, algo que también ponen de manifiesto trabajos como el de Abad et al. (2008).

Del mismo modo, y respecto a los programas ofertados, se ha podido comprobar que reconociendo la diversidad entre universidades (Alcedo et al., 2007), los ámbitos de intervención son bastante comunes, como ya constató en su estudio Alcantud et al. (2000), centrándose en programas de apoyo técnico, de formación dirigidos a toda la comunidad universitaria y de atención individualizada, temáticas que están en consonancia con las líneas de actuación desarrolladas en los servicios y con las finalidades que en ellos se persiguen.

Todo ello parece apuntar, en suma, en una misma dirección, esto es, que las instituciones de educación superior aúnen esfuerzos en proporcionar soporte a los/as estudiantes con discapacidad para que puedan concluir con éxito sus estudios y se adapten al contexto universitario (Sakiz y Saricali, 2017).

En conclusión, teniendo en cuenta lo hasta aquí expuesto, cabe señalar que, sin obviar las necesarias acciones relacionadas con la sensibilización, información, formación y mejora de la accesibilidad (adecuación de espacios, eliminación de barreras arquitectónicas, de comunicación, ...), parece necesario reforzar las actuaciones de estos servicios, centrando los esfuerzos en la orientación académica (flexibilización y adaptaciones curriculares, etc.), personal (actividades culturales, deportivas, participación estudiantil, etc.) y profesional (adquisición y desarrollo de habilidades sociolaborales, búsqueda de empleo, etc.) y prestar una adecuada atención personalizada mediante planes de acción tutorial. 
Finalmente, decir que este estudio se ha visto condicionado por una serie de limitaciones entre las que destacan, fundamentalmente, la no inclusión de datos sobre determinadas características de estos servicios que pueden aportar información útil sobre los mismos, como por ejemplo, el número de profesionales con los que cuentan, el modelo de intervención que siguen, su grado de especialización, los recursos (económicos, materiales) disponibles, la normativa específica y los protocolos con que cuenta cada universidad, aspectos que se erigen como posibles futuras líneas de indagación.

Asimismo, para el avance en la investigación sobre esta temática sería deseable analizar universidades de otros contextos, lo que permitiría, por una parte, conocer la situación específica de estas instituciones, las políticas que siguen en torno a la atención del alumnado con discapacidad y, por otra parte, avanzar en el conocimiento de las diferencias entre nuestra realidad y la de otros países, para determinar los cambios y mejoras que podrían incorporarse en las políticas organizativas y de orientación y tutoría de nuestras universidades.

\section{Referencias bibliográficas}

Abad, Ma․ Álvarez, P., y Castro de Paz, J. F. (2008). Apoyo a la integración de estudiantes con discapacidad en la enseñanza universitaria. Algunas medidas y propuestas de actuación orientadora. Educación y diversidad= Education and diversity: Revista inter-universitaria de investigación sobre discapacidad e interculturalidad, 2, 129-150.

Alcantud, F., Ávila, V., y Asensi, C. (2000). Adaptaciones curriculares en los estudios superiores. Minusval, 125, 17-19.

Álvarez Pérez, P., Alegre, O., y López, D. (2012). Las dificultades de adaptación a la enseñanza universitaria de los estudiantes con discapacidad: un análisis desde un enfoque de orientación inclusiva. Relieve, Revista Electrónica de Investigación y Evaluación Educativa, 18 (2). https://doi.org/10.7203/relieve.18.2.1986

Bausela Herreras, E. (2002). Atención a la diversidad en educación superior. Profesorado: Revista de currículum y formación del profesorado, 6 (1-2), 1-11. https://recyt.fecyt.es/index.php/profesorado/article/view/41971/23954

Bilbao, M. C. (2010). Percepción de los recursos que favorecen la integración de estudiantes con discapacidad en la educación superior según los docentes de la Universidad de Burgos. Educación y Diversidad, 4(2), 33-50.

Cotán, A. (2017). Educación inclusiva en las instituciones de educación superior: narrativas de estudiantes con discapacidad. Revista Española de Discapacidad, 5(1), 43-61.

Comes, G., Parera, B., Vedriel, G., y Vives, M. (2011). La inclusión del alumnado con discapacidad en la universidad: la opinión del profesorado. Innovación Educativa, 21, 173183.

De Los Santos, S. B., Kupezynski, L., y Mundy, M-A. (2019). Determining academic success in students whit disabilities in higher education. International Journal of Higher Education, 8(2), 16-38.

Fundación Universia (2018). Universidad y Discapacidad. IV Estudio sobre el grado de inclusión del sistema universitario español respecto de la realidad de la discapacidad. 
https://www.fundacionuniversia.net/wpcontent/uploads/2019/05/IVEstudio_UniversidadyDiscapacidad_ACC.pdf

Gairín, J. Muñoz, J. L., Feixas M., y Guillamón, C. (2009). La transición secundaria-universidad y la incorporación a la universidad. La acogida de los estudiantes de primer curso. Revista Española de Pedagogía, 242, 27-44.

Galán-Mañas, A. (2015). Orientación a los estudiantes con discapacidad en la Universidad Española. Revista de Orientación Psicopedagógica, 1 (26), 83-99. https://doi.org/10.5944/reop.vol.26.num.1.2015.14344

García-Cano, M., Castillejo, A., Jiménez, N., y Martínez, I. (2017). Universidad y discapacidad. Diagnóstico sobre la inclusión de estudiantes con discapacidad en la Universidad de Córdoba. Docencia y Derecho, Revista para la docencia jurídica universitaria, 11, 2-17.

Hernández, C. M., Fernández Mํㅡㄹ del M., Carrión, J. J., y Avilés, B. (2019). Revista Complutense de Educación, 30(4),1097-1112. https://doi.org/10.5209/rced.60106

Ley 137/1982, de 7 de abril, de integración social de los minusválidos (LISMI). Boletín Oficial del Estado, 30 de abril de 1982, núm. 103, pp. 11106-11112. https://www.boe.es/boe/dias/1982/04/30/pdfs/A11106-11112.pdf

Ley 51/2003, de 2 de diciembre, de igualdad de oportunidades, no discriminación y accesibilidad universal de las personas con discapacidad. Boletín Oficial del Estado, 3 de diciembre de 2003, núm. 289, pp. 43187-43195. https://www.boe.es/boe/dias/2003/12/03/pdfs/A4318743195.pdf.

Ley 39/2006, de 14 de diciembre, de Promoción de la Autonomía Personal y Atención a las personas en situación de dependencia. Boletín Oficial del Estado, 15 de diciembre de 2006, núm. 299, pp. 44142-44156. https://www.boe.es/boe/dias/2006/12/15/pdfs/A4414244156.pdf

Ley 49/2007, de 26 de diciembre, por la que se establece el régimen de infracciones y sanciones en materia de igualdad de oportunidades, no discriminación y accesibilidad universal de las personas con discapacidad. Boletín Oficial del Estado, 27 de diciembre de 2007, núm. 310, pp. 53278-53284. https://www.boe.es/buscar/pdf/2007/BOE-A-2007-22293-consolidado.pdf

Ley Orgánica 6/2001, de 21 de diciembre, de Universidades. Boletín Oficial del Estado, 21 de diciembre de 2001, núm. 307, pp. 49400-49425. https://www.boe.es/boe/dias/2001/12/24/pdfs/A49400-49425.pdf.

Ley Orgánica 4/2007, de 12 de abril, por la que se modifica la Ley Orgánica 6/2001, de 21 de diciembre, de Universidades. Boletín Oficial del Estado, 13 de abril de 2007, núm. 89, pp. 16241-16260. https://www.boe.es/boe/dias/2007/04/13/pdfs/A16241-16260.pdf.

Melero, N., Moriña, A., y Perera, V.-H. (2019). Acciones del profesorado para una práctica inclusiva en la universidad. Revista Brasileira de Educaçao, 24, 1-19. http://dx.doi.org/10.1590/s1413-24782019240016.

Molina, C., y González-Badía, J. (2006). Universidad y discapacidad: Guía de recursos. Cinca.

Núñez Mayán, M. T. (2017). Estudiantes con discapacidad e inclusión educativa en las universidades. Avances y retos para el futuro. Revista de Educación Inclusiva, 10 (1), 1330. https://revistaeducacioninclusiva.es/index.php/REl/article/view/269/0

ONU (2006). Convención Internacional de los Derechos de las Personas con Discapacidad. https://www.discapnet.es/areas-tematicas/nuestros-derechos/tus-derechosfondo/convencion-internacional. 
Peralta, A. (2007). Libro blanco sobre universidad y discapacidad. Real Patronato sobre discapacidad.

https://sapdu.unizar.es/sites/default/files/080326\%200856\%20Libro\%20Blanco.pdf

Real Decreto 743/2003, de 20 de junio, por el que se regula la prueba de acceso a la universidad de los mayores de 25 años. Boletín Oficial del Estado, 4 de julio de 2003, núm.159, pp. 26037-26039. https://www.boe.es/boe/dias/2003/07/04/pdfs/A26037-26039.pdf

Real Decreto 1742/2003, de 19 de diciembre, por el que se establece la normativa básica para el acceso a los estudios universitarios de carácter oficial. Boletín Oficial del Estado, 22 de $\begin{array}{lllll}\text { enero de } 2004, \quad \text { núm. } & \text { 2663-2667. }\end{array}$ https://www.boe.es/boe/dias/2004/01/22/pdfs/A02663-02667.pdf

Real Decreto 1393/2007, de 29 de octubre, por el que se establece la ordenación de las enseñanzas universitarias oficiales. Boletín Oficial del Estado, 30 de octubre de 2007, núm. 260, pp. 44037-44048. https://www.boe.es/boe/dias/2007/10/30/pdfs/A44037-44048.pdf.

Real Decreto 1791/2010, de 30 de diciembre, por el que se aprueba el Estatuto del Estudiante Universitario. Boletín Oficial del Estado, 31 de diciembre de 2010, núm. 318, pp. 109353109380. https://www.boe.es/boe/dias/2010/12/31/pdfs/BOE-A-2010-20147.pdf.

Real Decreto Legislativo 1/2013, de 29 de noviembre, por el que se aprueba el Texto Refundido de la Ley General de Derechos de las Personas con Discapacidad y de su inclusión social. Boletín Oficial del Estado, 3 de diciembre de 2013, núm. 289. http://noticias.juridicas.com/base_datos/Privado/517635-rdleg-1-2013-de-29-nov-seaprueba-el-texto-refundido-de-la-ley-general-de.html.

Red-SAPDU. https://sapdu.unizar.es/

Sakiz, H., y Saricali, M. (2017). Including students with visual difficulty within higher education: necessary steps. Exceptionality, A Special Education Journal, 26 (4), 266-282. https://doi.org/10.1080/09362835.2017.1283627

Suárez L. B., y Castillo, I. (2020). Descripción de una experiencia educativa inclusiva con alumnado universitario: Trabajando habilidades para el empleo. Tendencias Pedagógicas, 35, 130-152. doi:10.15366/tp2020.35.001

Vieira, M. J., y Ferreira, C. (2011). Los servicios de atención a estudiantes con discapacidad en las Universidades de Castilla y León. Revista Española de Orientación y Psicopedagogía, 22(2), 185-199. https://doi.org/10.5944/reop.vol.22.num.2.2011.68

Fecha de entrada: 17 noviembre 2019

Fecha de revisión: 8 septiembre 2020

Fecha de aceptación: 23 septiembre 2020 